\title{
Comparing oncologic outcomes in patients undergoing surgery for oncocytic neoplasms, conventional oncocytoma, and chromophobe renal cell carcinoma
}

Chandra K. Flack MDa, Adam C. Calaway MD, MPHa, Brady L. Miller MDº, Maria M. Picken MD, PhDc, Dibson D. Gondim MDd, Muhammad T. Idrees MBBS ${ }^{d}$, E. Jason Abel MD, FACS ${ }^{b}$, Gopal N. Gupta MD, FACS ${ }^{e}$, Ronald S. Boris MDa.
a. Departments of Urology, Indiana University School of Medicine, Indianapolis, IN
b. Department of Urology, University of Wisconsin School of Medicine, Madison, WI
c. Departments of Pathology, Loyola University School of Medicine, Maywood, IL
d. Departments of Pathology and Laboratory Medicine, Indiana University School of Medicine, Indianapolis, IN
e. Departments of Urology, Loyola University School of Medicine, Maywood, IL

\begin{abstract}
\section{INTRODUCTION}

Oncocytic neoplasms are renal tumors similar to oncocytoma, but their morphologic variations preclude definitive diagnosis. This somewhat confusing diagnosis can create treatment and surveillance challenges for the treating urologist. We hypothesize that these subtle morphologic variations do not drastically affect the malignant potential of these tumors, and we sought to demonstrate this by comparing clinical outcomes of oncocytic neoplasms to those of classic oncocytoma and chromophobe.
\end{abstract}

\section{METHODS}

We gathered demographic and outcomes data for patients with variant oncocytic tumors. Oncologic surveillance was conducted per institutional protocol in accordance with NCCN guidelines. Descriptive statistics were used to compare incidence of metastasis and death against those for patients with oncocytoma and chromophobe. Three hundred and fifty-one patients were analyzed: 164 patients with oncocytoma, 28 with oncocytic neoplasms, and 159 with chromophobe tumors.

\section{RESULTS}

Median follow-up time for the entire cohort was 32.4 months, (interquartile range 9.2-70.0). Seventeen total patients $(17 / 351,4.9 \%)$ died during the course of the study. In patients with 
oncocytoma or oncocytic neoplasm, none were known to metastasize or die of their disease. Only chromophobe tumors $>6 \mathrm{~cm}$ in size in our series demonstrated metastatic progression and approximately half of these metastasized tumors demonstrated sarcomatoid changes.

\section{CONCLUSION}

Variant oncocytic neoplasms appear to have a natural course similar to classic oncocytoma. These tumors appear to have no metastatic potential, and oncologic surveillance may not be indicated after surgery.

Keywords: Kidney, Nephrectomy, Neoplasms, Pathology.

\section{INTRODUCTION}

Oncocytomas are largely thought to be benign renal tumors with minimal malignant potential, representing $3 \%$ to $7 \%$ of all renal tumors and $15 \%$ of tumors $4 \mathrm{~cm}$ or less [1], [2], [3]. Diagnosis is easy when conventional morphologic features are present; however, variant oncocytic neoplasms can exhibit tremendous morphologic variations with overlapping histologic features and deviant immunohistochemical characteristics. The diagnostic difficulty due to these factors leads to designation with unconventional nomenclature including "borderline oncocytic neoplasm," "unclassified tumor with oncocytic features," or "hybrid oncocytic." It is estimated that $5 \%$ of all oncocytomas are these rare oncocytic tumors with unconventional features [4]. Unlike pure oncocytomas, the malignant potential of these variant oncocytic tumors is unknown creating treatment and surveillance dilemmas for the practicing urologist.

In our current study, we sought to examine the natural history of patients diagnosed with variant oncocytic neoplasms after surgical extirpation. We elected to compare the malignant potential (recurrence patterns) and overall survival of these variant oncocytic neoplasms with oncocytomas and chromophobe renal cell carcinoma (RCC) due to similar histologic attributes. We hypothesized that the recurrence and survival of patients with variant oncocytic neoplasms would be similar, regardless of pathologic description, to patients with pure oncocytoma and small indolent chromophobe tumors.

\section{MATERIALS AND METHODS}


We retrospectively identified all patients who underwent partial or radical nephrectomy at 3 academic institutions (University of Wisconsin, Loyola University, and Indiana University) between 2000 and 2016 for pathologically confirmed oncocytoma and variant oncocytic tumors which are referred to herein as "oncocytic neoplasms." This heterogenous group consisted of patients with the diagnosis of "borderline oncocytic neoplasm," "unclassified tumor with oncocytic features" or "hybrid oncocytic." Patients diagnosed with chromophobe renal cell carcinoma were also identified and outcomes were compared with the other groups due to similar histologic features and presumed indolent course. Pathologic processing of these tumors is described in the paragraphs following, with photomicrographs as shown in Fig. 1. Patients with prior history or concurrent renal neoplasms of another histologic subtype (clear cell, papillary, and urothelial) or those whose tumors were secondary to a known hereditary syndrome were excluded.

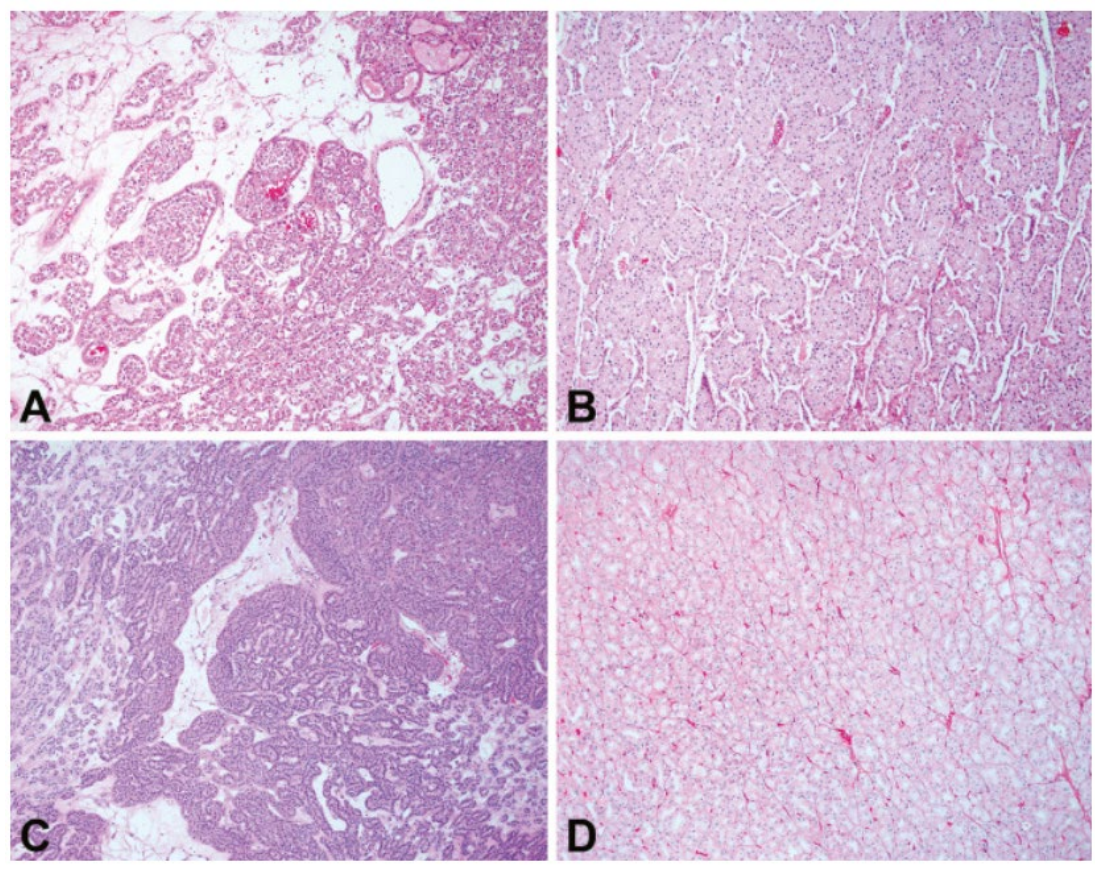

Fig. 1. A. Oncocytoma. B. Chromophobe RCC. C. Oncocytic neoplasm. D. RCC unclassified with oncocytic features.

Demographic and surgical data, as well as the clinical outcomes of metastasis and death were collected for each patient. Although not controlled for across institutions, patients diagnosed with malignant neoplasms had surveillance/imaging based on NCCN 
guidelines. Patients with oncocytoma were not routinely imaged per protocol. All patients with oncocytic neoplasms had surveillance imaging during follow-up, at the discretion of the practitioner, but this was not standardized among groups.

\subsection{TUMOR CATEGORIES: ONCOCYTOMA}

Morphology is the gold standard for establishing the diagnosis of oncocytoma. Conventional microscopic features include a central scar, solid or solid nested architecture, bland nuclei, abundant eosinophilic granular cytoplasm, loose hypocellular stroma, and circumscribed contours are observed. Occasionally, oncocytomas demonstrate unusual morphologic patterns including diffuse, papillary or solid sheet-like architecture, atypical nuclear features, lack of circumscription, encroachment into hilar or perirenal fat, and rarely the presence of cells in vascular spaces. Immunohistochemical stains are extremely helpful in confirming the diagnosis.

\subsection{TUMOR CATEGORIES: ONCOCYTIC NEOPLASMS}

Cases with unconventional features that are difficult to characterize are not designated as benign or malignant. These are labeled as "oncocytic renal neoplasm" with additional clarifying comments. Several terminologies including "low-grade," "borderline features," "unclassified," "low malignant potential," and "uncertain malignant potential," have been used to further classify these; however, so far no unifying nomenclature has been proposed [5]. Renal cell carcinoma unclassified is also included in this category when a major proportion of the tumor comprises of eosinophilic cells. These tumors could not be categorized based on distinct morphologic and immunohistochemical characteristics.

\subsection{TUMOR CATEGORIES: CHROMOPHOBE RCC}

Chromophobe RCC displays tumor cells arranged in solid sheets with hyalinized vascular septae. Other less common patterns may be encountered including tubular, small nest, papillary, trabecular, and microcystic. The predominant cells are large with pale reticular

cytoplasm and prominent plant-like cell membranes. The nuclei are hyperchromatic and wrinkled to resinoid with perinuclear clearing (haloes).

\subsection{STATISTICAL ANALYSIS}


Descriptive statistics were used to compare characteristics of patients with oncocytoma, oncocytic neoplasms, and chromophobe tumors. Analysis of variance was used to compare continuous variables, and Fishers Exact and Chi-squared tests, where appropriate, were used to compare categorical variables. Instances of metastasis and death were tallied for each category. Subgroup analysis was performed for tumors less than and $>7 \mathrm{~cm}$.

\section{RESULTS}

Three hundred and fifty-one patients were included in this analysis: 164 patients with oncocytoma, 28 with oncocytic neoplasms, and 159 with chromophobe tumors. Median follow-up time for the entire cohort was 32.4 months, (interquartile range [IQR] 9.2-70.0). Follow-up for each group was as follows: 33.3 months (IQR 6.4-70.2) for oncocytoma, 42.0 months (IQR 23.1-62.0) for oncocytic neoplasms, and 29.6 months (IQR 12.6-70.4) for chromophobe $(P=0.522)$. Patient demographic, surgical type, and tumor characteristics are described in Table 1. 
Table 1. Patient demographics and tumor characteristics

\begin{tabular}{|c|c|c|c|c|}
\hline & $\begin{array}{l}\text { Oncocytoma } \\
(164)\end{array}$ & $\begin{array}{l}\text { Oncocytic neoplasm } \\
(28)\end{array}$ & $\begin{array}{l}\text { Chromophobe } \\
\text { (159) }\end{array}$ & $P$ value \\
\hline Age, y (mean, SD) & $65.0(9.8)$ & $67.0(15.7)$ & $53.4(14.5)$ & $<0.0001^{a}$ \\
\hline Sex (male) & $109(66.5 \%)$ & $15(53.6 \%)$ & $88(55.4 \%)$ & $0.092^{b}$ \\
\hline Race (nonwhite) & $12(7.3 \%)$ & $4(14.3 \%)$ & $17(10.6 \%)$ & $0.381^{b}$ \\
\hline Surgery type & & & & $<0.001^{b}$ \\
\hline Radical & $50(30.5 \%)$ & $13(46.4 \%)$ & $93(58.5 \%)$ & \\
\hline Partial & $114(69.5 \%)$ & $15(53.6 \%)$ & $66(40.2 \%)$ & \\
\hline Tumor size, cm. (mean, SD) & $3.5(2.3)$ & $4.4(3.0)$ & $6.0(4.8)$ & $<0.0001^{a}$ \\
\hline Tumor stage & & & & $<0.0001^{b}$ \\
\hline T1a & $118(72.0)$ & $15(53.6)$ & $72(45.3)$ & \\
\hline T1b & $36(22.0)$ & $5(17.9)$ & $38(23.9)$ & \\
\hline T2a & $6(3.7)$ & $2(7.1)$ & $18(11.3)$ & \\
\hline $\mathrm{T} 2 \mathrm{~b}$ & $3(1.8)$ & $0(0)$ & $13(8.2)$ & \\
\hline T3a & $1(0.6)$ & $6(21.4)$ & $18(11.3)$ & \\
\hline
\end{tabular}

$\mathrm{IQR}=$ interquartile range.

a Statistical tests used: Student's $t$ test.

${ }^{\mathrm{b}}$ Fischer's Exact test.

\subsection{ONCOCYTOMA AND ONCOCYTIC NEOPLASMS}

Overall survival for patients was 95.7\% (157/164) for oncocytoma and 93.9\% (26/28) for oncocytic neoplasms $(P=0.136)$. In patients possessing oncocytoma or an oncocytic neoplasm, none were known to metastasize and disease-specific survival in this group was $100 \%$. Analysis of tumors less than $7 \mathrm{~cm}$ in size showed survival of $95.4 \%(146 / 153)$ for oncocytoma, $91.3 \%(21 / 23)$ for oncocytic neoplasm.

\subsection{CHROMOPHOBE}


Nine patients, all chromophobe, experienced metastasis (9/159, 5.7\%), and 3 of those patients died of their disease (3/159, 1.8\%). Overall survival was $94.9 \%(150 / 159)$ for chromophobe tumors $(P=0.136)$. Two deaths occurred secondary to disease recurrence after nephrectomy, and one death was related to metastasis at presentation. Of the 9 patients that developed metastatic disease, 4 of their tumors exhibited sarcomatoid components $(4 / 9,44.4 \%$ ) and all were large (ranging from 6 to $21.7 \mathrm{~cm}$ in greatest dimension). Analysis of chromophobe tumors less than $7 \mathrm{~cm}$ in size showed overall survival of $95.5 \%$ (107/113). For size $\geq 7$, overall survival was $95.7 \%(44 / 46)$.

\section{DISCUSSION}

Oncocytoma shares similarities in morphology with other eosinophilic tumors of the kidney. These tumors have been postulated to stem from a similar line of differentiation and exist on a spectrum of increasing risk of malignancy rather than as distinct entities [6]. We hypothesized that intricate differentiation between the various oncocytic tumors does not impact overall prognosis, and that once highly malignant characteristics are excluded, these tumors may be followed similarly. Data presented here support the hypothesis that oncocytic neoplasms behave more like oncocytoma than chromophobe RCC. These oncocytic neoplasms are biologically distinct from other "unclassified" renal masses that may possess high-risk pathologic features and a more worrisome fate.

Published literature supporting the benign nature of classic oncocytoma is consistent with the results in our present study. A European multicenter retrospective review of 32 cases of oncocytoma revealed no incidence of metastasis in their cohort. After 54 months, all of these patients exhibited $100 \%$ disease-specific survival. In a second study by Dechet et al., 138 patients with oncocytoma were followed for a mean duration of 41 months. Metastatic-free and disease-specific survival were 100\% [7]. Death from progression of oncocytoma following surgery has been effectively ruled out from a surplus of publications over the past 2 decades [8], [9], [10], [11]. As data amasses to support the benign nature of these tumors, published guidelines now support minimal treatment and surveillance of proven oncocytoma. The American Urological Association guidelines identify oncocytoma as a benign lesion and European Association of Urology now advocates that oncocytomas, when histologically confirmed, can be safely observed [12], [13]. We 
believe that these guidelines may be extended to patients with bilateral synchronous masses, no evidence of a genetic syndrome such as Birt-Hogg-Dube, or a known diagnosis of an oncocytoma or oncocytic neoplasm. In cases of bilateral renal masses and a known diagnosis of oncocytoma, pathologic concordance with the contralateral side has been reported to be as high as $94 \%$ [14].

While most chromophobe tumors carry a good prognosis compared with clear cell RCC, metastasis and death are still encountered, particularly with large tumors and/or those with sarcomatoid components [15], [16]. In a pooled analysis, the rate of metastasis in 878 patients with chromophobe tumors was $7 \%$ which was comparable to rate reported in our current study [17]. It is reasonable to remain vigilant against the possibility of mistaking chromophobe RCC as a benign entity. As such, some practitioners may be tempted to pursue more aggressive surveillance strategies when pathologic reporting is equivocal (as is in the case of the diagnosis of "oncocytic neoplasm"). Our study suggests that these histologic nuances may be less important than more objective data, such as tumor size and the presence or absence of sarcomatoid features which have been repeatedly shown to more strongly affect prognosis. Specifically, only tumors $>6 \mathrm{~cm}$ in size in our series demonstrated any metastatic progression and approximately half of these metastasized tumors demonstrated sarcomatoid changes. These results suggest a minimized postoperative surveillance protocol is likely safe for oncocytic neoplasms without high-risk features. As active surveillance develops a clear role in appropriately selected patients and the utilization of renal mass biopsy increases, investigating the role of a nonoperative approach for oncocytic neoplasms is warranted and appears to be an appropriate future direction of study.

Several limitations to this study should be mentioned. The study was retrospective and follow-up was short. Although clinical guidelines were followed for all institutions, followup was not standardized for purposes of protocol. Due to the scarcity of oncocytic neoplasms, the number of analyzed tumors in this category was fewer than with oncocytoma or chromophobe renal cell carcinoma. Despite the inclusion of multiple institutions, nondiagnostic oncocytic neoplasms were still infrequently encountered, limiting the generalizability of our results. Furthermore, large tumors and tumors with high- 
risk features were underrepresented in our cohort. Explanations for the vast majority of oncocytic neoplasms having lower risk features may be multifactorial. It is possible that they were simply not identified in our pathologic review or that some high-risk features may have effectively "ruled out" pathologic inclusion as a oncocytic neoplasm and were therefore not included in our analysis. It is also possible that due to inherently slower growth kinetics they are more likely to be found at a smaller size. Follow-up may be warranted to look only at T2 oncocytic neoplasms from a larger multi-institutional review to evaluate their biologic effect. Lack of central pathology across institutions may have allowed for some degree of interobserver variability when categorizing the tumors into each histopathologic category. Paradoxically, this strengthens our message that the individual pathologic nuances of these tumors may be less important as the overwhelming majority do not recur or metastasize. These limitations notwithstanding, our study represents the largest cohort study evaluating the biologic behavior of all subtypes of renal oncocytoma and the first to underscore the low aggressive behavior of oncocytic neoplasms.

\section{CONCLUSIONS}

Provided that appropriate exclusion criteria are used, oncocytic neoplasms appear to represent an indolent subset of renal masses with a short-term prognosis similar to classic oncocytoma. The presence of oncocytic features on final pathology should reassure the provider of a less aggressive tumor biology requiring a less stringent follow-up surveillance strategy. Chromophobe renal cell carcinoma, alternatively, especially when large or having high-risk pathologic features, can metastasize and does continue to warrant close observation following surgical removal. 


\section{REFERENCES}

1. Haifler, M., Copel, L., Sandbank, J., Lang, E., Raz, O., Leibovici, D., Lindner, A., \& Zisman, A. (2012). Renal oncocytoma-Are there sufficient grounds to consider surveillance following prenephrectomy histologic diagnosis. Urologic Oncology: Seminars and Original Investigations, 30(4), 362-368. https://doi.org/10.1016/j.urolonc.2009.11.024

2. Humphrey, P. A. (2012). Oncocytoma of the Kidney. The Journal of Urology. https://www.auajournals.org/doi/abs/10.1016/j.juro.2012.02.007

3. Ricketts, C. J., \& Maher, E. R. (2015). Renal Cell Carcinoma: Overview. In V. H. Nargund, D. Raghavan, \& H. M. Sandler (Eds.), Urological Oncology (pp. 337344). Springer. https://doi.org/10.1007/978-0-85729-482-1_17

4. P. Austin, G. Vricella, M. Campdell Campbell-Walsh urology (2016)

5. Williamson, S. R., Gadde, R., Trpkov, K., Hirsch, M. S., Srigley, J. R., Reuter, V. E., Cheng, L., Kunju, L. P., Barod, R., Rogers, C. G., Delahunt, B., Hes, O., Eble, J. N., Zhou, M., McKenney, J. K., Martignoni, G., Fleming, S., Grignon, D. J., Moch, H., \& Gupta, N. S. (2017). Diagnostic criteria for oncocytic renal neoplasms: A survey of urologic pathologists. Human Pathology, 63, 149-156. https://doi.org/10.1016/j.humpath.2017.03.004

6. Algaba, F., Akaza, H., López-Beltrán, A., Martignoni, G., Moch, H., Montironi, R., \& Reuter, V. (2011). Current Pathology Keys of Renal Cell Carcinoma. European Urology, 60(4), 634-643. https://doi.org/10.1016/j.eururo.2011.06.047

7. Dechet Christopher B., Bostwick David G., Blute Michael L., Bryant Sandra C., \& Zincke Horst. (1999). Renal oncocytoma: Multifocality, bilateralism, metachronous tumor development and coexistent renal cell carcinoma. Journal of Urology, 162(1), 40-42. https://doi.org/10.1097/00005392-199907000-00010

8. DAVIS, C. (1991). Renal oncocytoma. Clinicopathological study of 166 patients. J. Urogenital. Pathol., 1, 41-52.

9. Frydenberg, M., Eckstein, R. P., Saalfield, J. a. a. H., Breslin, F. H. D., Alexander, J. H., \& Roche, J. (1991). Renal Oncocytomas-An Australian Experience. British Journal of Urology, 67(4), 352-357. https://doi.org/10.1111/j.1464410X.1991.tb15160.x 
10.Amin, M. B., Crotty, T. B., Tickoo, S. K., \& Farrow, G. M. (1997). Renal Oncocytoma: A Reappraisal of Morphologic Features with Clinicopathologic Findings in 80 Cases. The American Journal of Surgical Pathology, 21(1), 1-12.

11. Gudbjartsson, T., Hardarson, S., Petursdottir, V., Thoroddsen, A., Magnusson, J., \& Einarsson, G. V. (2005). Renal oncocytoma: A clinicopathological analysis of 45 consecutive cases. BJU International, 96(9), 1275-1279. https://doi.org/10.1111/j.1464-410X.2005.05827.x

12. Ljungberg, B., Cowan, N. C., Hanbury, D. C., Hora, M., Kuczyk, M. A., Merseburger, A. S., Patard, J.-J., Mulders, P. F. A., \& Sinescu, I. C. (2010). EAU Guidelines on Renal Cell Carcinoma: The 2010 Update. European Urology, 58(3), 398-406. https://doi.org/10.1016/j.eururo.2010.06.032

13. Campbell Steven, Uzzo Robert G., Allaf Mohamad E., Bass Eric B., Cadeddu Jeffrey A., Chang Anthony, Clark Peter E., Davis Brian J., Derweesh Ithaar H., Giambarresi Leo, Gervais Debra A., Hu Susie L., Lane Brian R., Leibovich Bradley C., \& Pierorazio Philip M. (2017). Renal Mass and Localized Renal Cancer: AUA Guideline. Journal of Urology, 198(3), 520-529. https://doi.org/10.1016/j.juro.2017.04.100

14. Boris, R. S., Benhammou, J., Merino, M., Pinto, P. A., Linehan, W. M., \& Bratslavsky, G. (2011). The Impact of Germline BHD Mutation on Histological Concordance and Clinical Treatment of Patients With Bilateral Renal Masses and Known Unilateral Oncocytoma. The Journal of Urology, 185(6), 2050-2055. https://doi.org/10.1016/j.juro.2011.02.051

15. Renshaw, A. A., Henske, E. P., Loughlin, K. R., Shapiro, C., \& Weinberg, D. S. (1996). Aggressive variants of chromophobe renal cell carcinoma. Cancer, 78(8), 1756-1761. https://doi.org/10.1002/(SICI)1097-0142(19961015)78:8<1756::AIDCNCR16>3.0.CO;2-X

16. Klatte, T., Han, K., Said, J. W., Böhm, M., Allhoff, E. P., Kabbinavar, F. F., Belldegrun, A. S., \& Pantuck, A. J. (2008). Pathobiology and prognosis of chromophobe renal cell carcinoma. Urologic Oncology: Seminars and Original Investigations, 26(6), 604-609. https://doi.org/10.1016/j.urolonc.2007.07.015 
17. Amin, M. B., Paner, G. P., Alvarado-Cabrero, I., Young, A. N., Stricker, H. J., Lyles, R. H., \& Moch, H. (2008). Chromophobe Renal Cell Carcinoma: Histomorphologic Characteristics and Evaluation of Conventional Pathologic Prognostic Parameters in 145 Cases. The American Journal of Surgical Pathology, 32(12), 1822-1834. https://doi.org/10.1097/PAS.0b013e3181831e68 